\title{
Comparative Study of Fast Superposition, Superposition Algorithm in Intensity Modulated Radiotherapy Techniques for Prostate Cancer
}

\author{
Mukesh Zope $^{1}$, Deepali Patil' ${ }^{1}$, Angel Kuriakose ${ }^{2}$, P. A. Aslam ${ }^{3}$, Basil George ${ }^{4}$, Abhijeet Mandal ${ }^{5}$ \\ ${ }^{1}$ Department of Medical Physics, State Cancer Institute, Indira Gandhi Institute of Medical Sciences, Patna, India \\ ${ }^{2}$ Department of Radiotherapy, Mahavir Cancer Sansthan, Patna, India \\ ${ }^{3}$ Speciality of Medical Physics, Super Speciality of Cancer Institute and Hospital, Lucknow, India \\ ${ }^{4}$ Department of Radiotherapy, Regional Cancer Care Center and Cancer Hospital, Siddhpur, India \\ ${ }^{5}$ Department of Radiotheraphy \& Radiation Medicine, Institute of Medical Sciences, Varansi, India \\ Email:mpdeepali23@gmail.com,zopeigims27@gmail.com, angel.tkm92@gmail.com, aslam.rhmn@gmail.com, \\ basilpgeorge92@yahoo.in, abhijit.mandal1@bhu.ac.in
}

How to cite this paper: Zope, M., Patil, D., Kuriakose, A., Aslam, P.A., George, B. and Mandal, A. (2020) Comparative Study of Fast Superposition, Superposition Algorithm in Intensity Modulated Radiotherapy Techniques for Prostate Cancer. International Journal of Medical Physics, Clinical Engineering and Radiation Oncology, 9, 62-72. https://doi.org/10.4236/ijmpcero.2020.92007

Received: March 17, 2020

Accepted: April 24, 2020

Published: April 27, 2020

Copyright $\odot 2020$ by author(s) and Scientific Research Publishing Inc. This work is licensed under the Creative Commons Attribution International License (CC BY 4.0).

http://creativecommons.org/licenses/by/4.0/

\begin{abstract}
This study aims to compare the dosimetric coverage of the Planning Target Volume (PTV) and the dose to main organs at risk (OARs) between two different algorithms fast superposition (FSUP), superposition (SUP) using intensity-modulated radiotherapy (IMRT) techniques for prostate cancer. Ten patients with prostate cancer were selected for this study. For each patient, IMRT plans were created with $6 \mathrm{MV}$ photon beam quality using CMS XiO treatment planning system. The delivery of IMRT was carried out using the step and shoot techniques. The dose coverage for each patient was designated to an ICRU report 62 reference point in the PTV, medium coverage of the planned target volume to be $95 \%$ of the prescribed dose while the maximum dose in the target volume to be not greater than $107 \%$ of the prescribed dose. A hypofractionated prescription dose of $70 \mathrm{~Gy} / 28 \#$ at $2.5 \mathrm{~Gy}$ per fraction was used. Besides we compared the number of MUs and OARs dose to D15\%, D25\%, D35\%, D50\% on both algorithm planning sets. For target, this evaluation was made with comparing the conformity index (CI) and homogeneity index (HI). In our study, the results show the OARs got less dose from the SUP algorithm compared to FSUP algorithms. Statistically not significant difference was observed in $\mathrm{V}_{107 \%}$ of PTV, MU/CC, conformity Index ( $\mathrm{p}=$ $0.057, p=0.215, p=0.370)$ and 95\% PTV Volume received prescription dose from both Plans. But Homogeneity Index for both algorithms was statistically significant $(p=0.000, p=0.001)$. For prostate cancer, the superposition algo-
\end{abstract}


rithm showed better results in the IMRT plan compared to the fast superposition algorithm.

\section{Keywords}

Prostate Cancer, IMRT, Algorithm, MUs, Conformity Index, Homogeneity Index

\section{Introduction}

Intensity Modulated Radiation Therapy (IMRT) techniques can provide better dose distribution for prostate cancer than manual 3DCRT planning. Intensitymodulated radiation therapy (IMRT) is one of the techniques which can deliver a radiation dose to the tumor in the form of sliding window (SW) or step and shoot (SS) methods [1]. IMRT increases the volume of normal tissue exposed to radiation but can reduce the total dose received by the organ a trisk [2], permits tumor dose escalation, thereby yielding higher rates of local tumor control [3] [4] [5]. Several single-institution series have reported are ductioninlate toxicity with the introduction of IMRT compared to 3DCRT, even with dose escalation [6].

The accuracy of dose calculation had been ameliorated by shifting from homogeneity corrections over pencil beam algorithms to point kernel-dependent Convolution/Superposition calculation algorithm [7].

In our study, Fast superposition, Superposition algorithms were used for each patient IMRT plan.

The purpose of this study was to compare the dosimetric coverage of the Planning Target Volume (PTV), the dose to main organs at risks (OARs) such as the rectum, bladder, femoral head, dose homogeneity index, conformity index and number of MUs between two different algorithms using intensity-modulated radiotherapy (IMRT) techniques for prostate cancer.

\section{Methods and Material}

Ten patients undergoing radical radiation treatment with histologically confirmed and clinically staged localized prostate cancer were selected for this study. The age of the patients ranged from 60 to 75 years. All patients were immobilized using the thermoplastic pelvic mask in the supine position with special instruction to keep their rectum empty and bladder comfortably fill at the time of CT simulation. Planning CT images with $3 \mathrm{~mm}$ thickness were acquired from the level of L2 - 3 to the ischialtuberosity. All CT images of the patient were transferred to the Elekta focal-SIM contouring workstation via DICOM for contouring.

Planning target volume and OARs such as the rectum, Bladder, Right \& Left femoral head were delineated as per RTOG guidelines [8].

The seven field $\left(25^{\circ}, 75^{\circ}, 130^{\circ}, 180^{\circ}, 230^{\circ}, 280^{\circ}, 335^{\circ}\right)$ IMRT plans were 
created using CMS XiO treatment planning system with $6 \mathrm{MV}$ photon beam and FSUP, SUP algorithm for each patient. Thus a total of 20 plans were generated using Step \& Shoot IMRT treatment delivery techniques with 80 leaf multileaf collimator and leaf width of $1 \mathrm{~cm}$ at the isocenter. A hypofractionated prescription dose of $70 \mathrm{~Gy} / 28 \#$ at $2.5 \mathrm{~Gy}$ per fraction was used.

Treatment planning was performed to achieve at least $95 \%$ of PTV volume receiving $95 \%$ of the Prescription dose and with less than $2 \%$ of PTV volume receiving $<107 \%$ of the prescribed dose.

For each plan, a dose-volume histogram (DVH) was generated using CMS XiO TPS. $\mathrm{D}_{\max }, \mathrm{D}_{\text {mean }}$, and $\mathrm{D}_{\text {min }}$ were recorded for PTV. HI, CI and the number of MUs were computed in all patients.

OARs such as rectum dose to D15\% $\geq 74 \mathrm{~Gy}, \mathrm{D} 25 \% \geq 69 \mathrm{~Gy}, \mathrm{D} 35 \% \geq 64 \mathrm{~Gy}$, $\mathrm{D} 50 \% \geq 59$ Gy and Bladder dose to D15\% $\geq 74$ Gy, D25\% $\geq 69$ Gy, D35\% $\geq 69$ Gy, D50\% $\geq 64$ Gy on both algorithm plan were computed. The femoral head kepta mean dose below $45 \mathrm{~Gy}$.

1) $D_{\max }$ : the absolute maximum dose received by any point in the OARs or PTV (inGy).

2) $D_{\text {min }}$ : the absolute minimum dose received by any point in the OARs or PTV (inGy).

3) $D_{\text {mean }}$ the absolute mean dose received by the OARs or PTV (inGy).

4) D15\%: the absolute dose received by the $15 \%$ of the OARs volume (inGy).

5) D25\%: the absolute dose received by the $25 \%$ of the OARs volume (inGy).

6) D35\%: the absolute dose received by the $35 \%$ of the OARs volume (inGy).

7) D50\%: the absolute dose received by the $50 \%$ of the OARs volume (inGy).

\section{Dosimetric plan evaluation parameters:}

To evaluate the dosimetric parameters, cumulative dose-volume histogram (DVHs) were calculated for each algorithm IMRT plan. To evaluate the target dose of each algorithm IMRT plan, homogeneity index (HI), conformity index (CI).

The homogeneity index (HI) was calculated according to the following formula [9]:

$$
\mathrm{HI}=(\mathrm{D} 2 \%-\mathrm{D} 98 \%) / \mathrm{D} 50 \%,
$$

where, D2, D98, and D50 represent the dose to $2 \%, 98 \%$, and $50 \%$ volume for the PTV, respectively.

Values of HI closer to 0 indicate greater dose homogeneity within the volume of PTV, while large values indicate more heterogeneous dose distribution.

The conformity index (CI) was calculated according to the RTOG protocol formula [10]:

$$
C I_{R T O G}=V_{R I} / T V
$$

where, $V_{R:}$ the volume of reference isodoseonbody, $T V$ : Total PTV Volume.

The closer value of CI to 1.0 the better the dose conformity.

Statistical analysis:

The difference between the two algorithm planning was compared using mean 
statistics for their radiated OARs volumes and PTV coverage as the main parameters. A paired t-test was used to verify the significance of the differences of the treatment plans, the $\mathrm{p}$-value of $\leq 0.05$ was taken into account as a significant difference.

\section{Results and Discussion}

\section{Comparison between the FSUP and SUP algorithms according to $D_{\max }$,} $\mathrm{D}_{\text {mean }}$ and $\mathrm{D}_{\min }$ doses for PTV:

Table 1 shows the maximum, mean and minimum doses for PTV IMRT plans were 76.66 70.85, 56.33 Gy for FSUP algorithm and 75.97, 70.59, 56.03 Gy for SUP algorithm.

However, the SP algorithm dose for maximum, mean, minimum is less than that of the FSP algorithm IMRT plan. Statistically, a significant difference was observed for both algorithm $(\mathrm{p}=0.004, \mathrm{p}=0.000, \mathrm{p}=0.007)$

Figures 1-3 shows the maximum value of average $D_{\max }, D_{\text {mean }}$, and $D_{\min }$ gets the lowest doses with the SUP algorithm compared the FSUP algorithm.

Comparison between the FSUP and SUP algorithms for PTV, HI, CI, MUs:

Compared to mean dosimetric parameters 95\% PTV volume received a dose to FSP algorithm was $66.63 \mathrm{~Gy}$ and 66.45 Gy for SUP algorithm IMRT plans. However, a statistically significant difference was observed for both Plans ( $\mathrm{p}=$ 0.0005) as shown in Table 2 \& Figure 4 shows a comparison between two algorithms.

Table 1. Comparison of $D_{\max }, D_{\text {mean }}, D_{\min }$. for PTV in both algorithm IMRT PLAN.

\begin{tabular}{|c|c|c|c|c|c|c|c|c|c|c|}
\hline \multirow{2}{*}{ PTV } & \multicolumn{4}{|c|}{ FSUP algorithm } & \multicolumn{4}{|c|}{ SUP algorithm } & \multicolumn{2}{|c|}{ PAIRED T TEST } \\
\hline & Mean & SD & Min. & Max. & Mean & SD & Min. & Max. & $\mathrm{t}$ & p-value \\
\hline$D_{\max }$ & 7665.98 & 174.19 & 7472 & 7961.7 & 7597 & 163.84 & 7430 & 7866 & 3.772 & $0.0044^{*}$ \\
\hline$D_{\text {mean }}$ & 7084.88 & 50.338 & 7013.9 & 7165.1 & 7058.8 & 45.979 & 6994.8 & 7132.4 & 10.894 & $0.0000^{*}$ \\
\hline$D_{\min }$ & 5633.39 & 436.36 & 4987 & 6494 & 5603.2 & 446.7 & 4926.5 & 6459.1 & 3.443 & $0.0074^{*}$ \\
\hline
\end{tabular}

${ }^{*}$ Significant difference from all $(\mathrm{p} \leq 0.05)$.

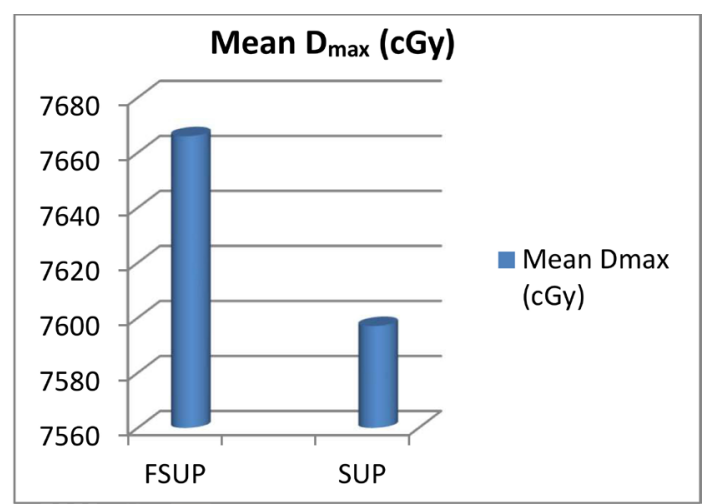

Figure 1. Comparison between two algorithms according to $D_{\max }$ for Prostate PTV with IMRT. 


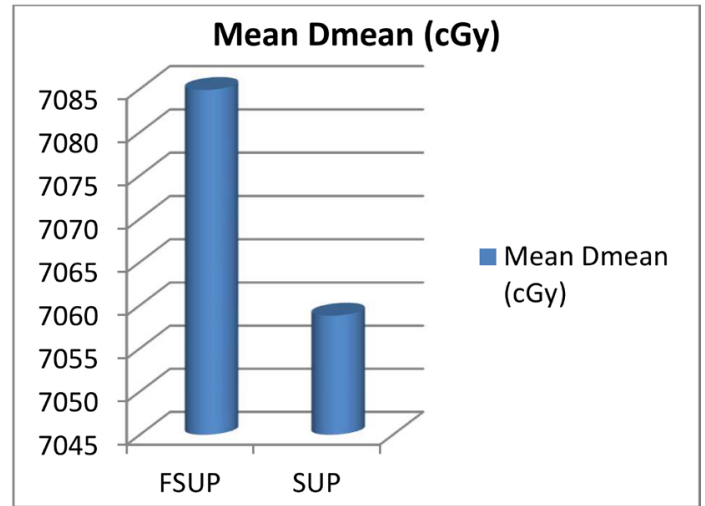

Figure 2. Comparison between two algorithms according to $\mathrm{D}_{\text {mean }}$ for Prostate PTV with IMRT.

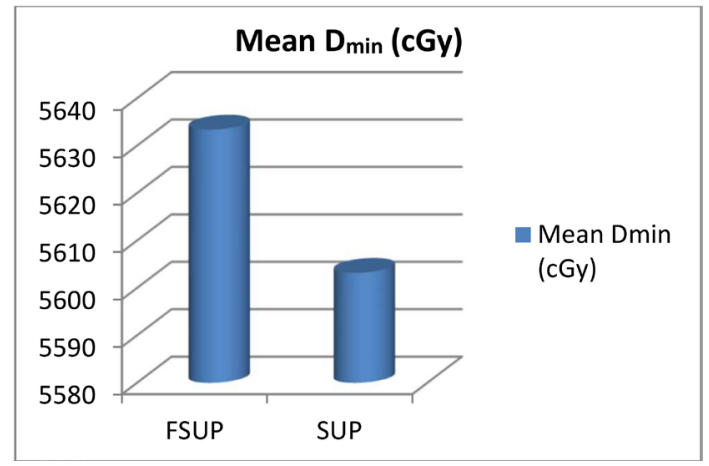

Figure 3. Comparison between two algorithms according to $\mathrm{D}_{\min }$ for Prostate PTV with IMRT.

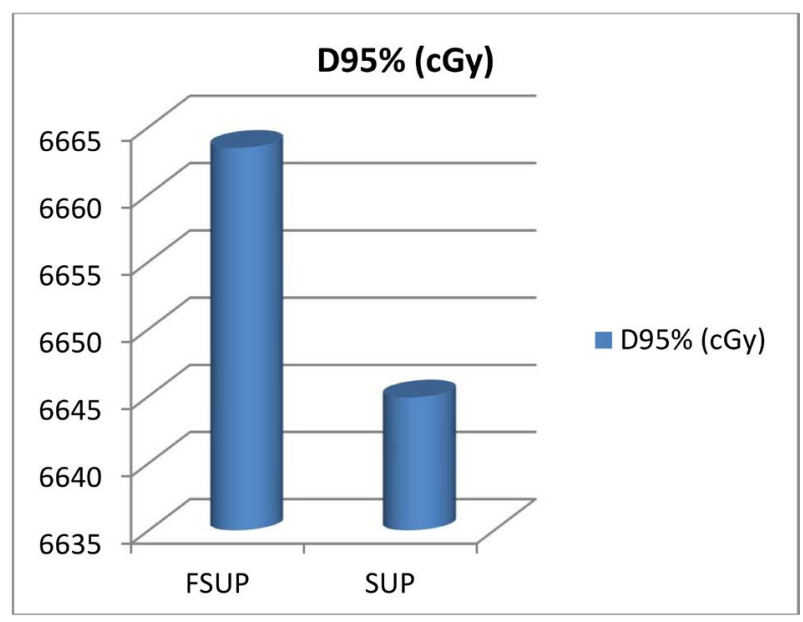

Figure 4. Comparison between two algorithms according to D95\% (cGy) for Prostate PTV with IMRT.

Compared to mean dosimetric parameters $107 \%$ prescription dose received volume to FSUP algorithm was $1.755 \%$ and $1.139 \%$ for SUP algorithm IMRT plans $(\mathrm{p}=0.0565)$. However, statistically not significant difference was observed in 107\% PTV volume for both algorithms as shown in Table 2 and Figure 5.

Figure 6 shows a comparison between two algorithms according to the average conformity indexes for PTV of prostate cancer patients with the IMRT plan. 
Table 2. Comparison of mean dosimetric parameters for PTV in both algorithm IMRT PLAN.

\begin{tabular}{|c|c|c|c|c|c|c|c|c|c|c|}
\hline \multirow{2}{*}{ PTV } & \multicolumn{4}{|c|}{ FSUP algorithm } & \multicolumn{4}{|c|}{ SUP algorithm } & \multicolumn{2}{|c|}{ PAIRED T TEST } \\
\hline & Mean & SD & Min. & Max. & Mean & SD & Min. & Max. & $\mathrm{t}$ & $\mathrm{p}$ \\
\hline $\mathrm{V}_{107 \%}$ & 1.755 & 3.3433 & 0.00 & 10.83 & 1.139 & 2.5258 & 0.00 & 8.2 & 2.187 & 0.0565 \\
\hline D95\% & 6663.45 & 131.87 & 6515.8 & 6907 & 6644.9 & 126.88 & 6502.1 & 6880.6 & 5.256 & $0.0005^{*}$ \\
\hline $\mathrm{CI}$ & 0.94967 & 0.0293 & 0.91453 & 0.99907 & 0.9473 & 0.0354 & 0.9091 & 1.0179 & 0.942 & 0.3706 \\
\hline HI & 0.10027 & 0.0248 & 0.05606 & 0.13292 & 0.0979 & 0.0245 & 0.0527 & 0.1296 & 4.634 & $0.0012^{*}$ \\
\hline MU/CC & 2.19905 & 1.4821 & 1.101 & 6.15942 & 2.1521 & 1.4843 & 1.1075 & 6.181 & 1.335 & 0.2147 \\
\hline
\end{tabular}

${ }^{*}$ Significant difference from all $(\mathrm{p} \leq 0.05)$.

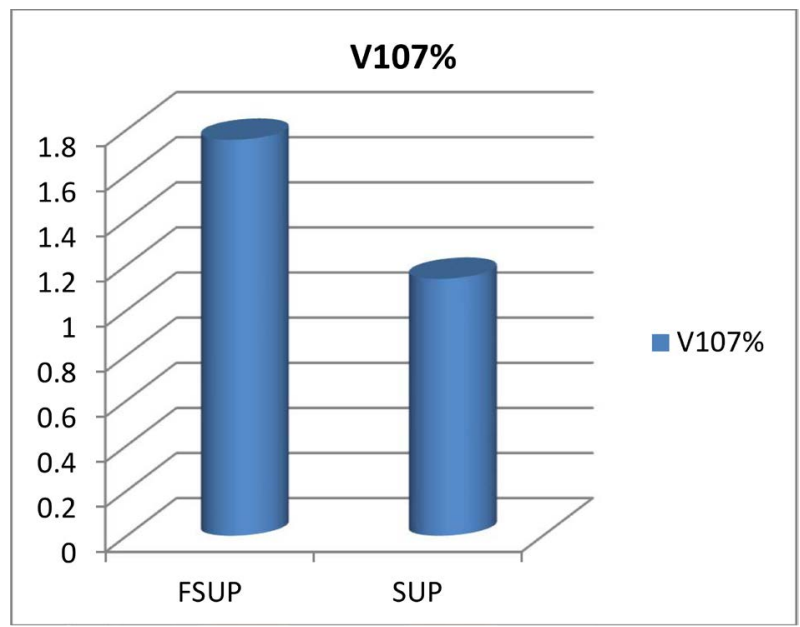

Figure 5. Comparison between two algorithms according to $\mathrm{V}_{107 \%}$ for Prostate PTV with IMRT.

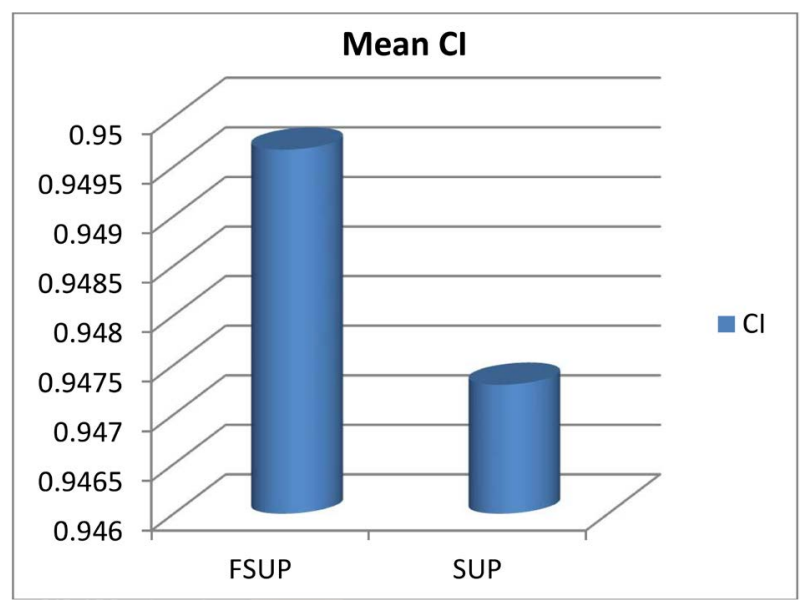

Figure 6. Comparison between two algorithms according to the conformity Index.

Conformity index PTV was 0.9496 for FSUP verses 0.9473 for SUP ( $p=0.370$ ) are not significant differences were observed in both algorithms in the IMRT plan as shown in Table 2.

SUP algorithm shows the minimum value of average HI (closer value to zero) and statistically significant differences were observed in both algorithms ( $\mathrm{p}=$ 
$0.001)$ as shown in Table 2 and Figure 7.

Compare to mean value MU/CC was 2.199 in FSUP and 2.152 in the SUP IMRT plan ( $\mathrm{p}=0.215)$. However, statistically not significant difference was observed as shown in Table $2 \&$ the number of monitor unit/CC gets lower with the SUP algorithm compared to the FSUP algorithm as shown in Figure 8.

Comparison between the FSUP and SUP algorithms for OARs (Rectum):

Figure $9 \&$ Table 3 shows the doses to 15\%, 25\%, 35\%, 50\% of the rectum volumes in two algorithms were 69.27 Gy, 66.17 Gy, 62.31 Gy, 55.67 Gy for FSP and 69.07 Gy, 65.99 Gy, 62.13 Gy, 55.49 Gy for SP algorithm for 7 field IMRT Plans. As we can see the dose of 15\%, 25\%, 50\% rectum volumes in the SP algorithm IMRT plan are less than that of the FSP algorithm.

However, rectum statistically not significant differences between the doses to $35 \%, 50 \%$ of the organ volumes $(\mathrm{p}=0.056, \mathrm{p}=0.173)$.

Comparison between the FSUP and SUP algorithms for OARs (Bladder):

Figure 10 \& Table 4 shows a Comparison between the doses to 15\%, 25\%, $35 \%, 50 \%$ of the bladder volumes were $70.78 \mathrm{~Gy}, 67.81 \mathrm{~Gy}, 62.90 \mathrm{~Gy}, 53.71 \mathrm{~Gy}$

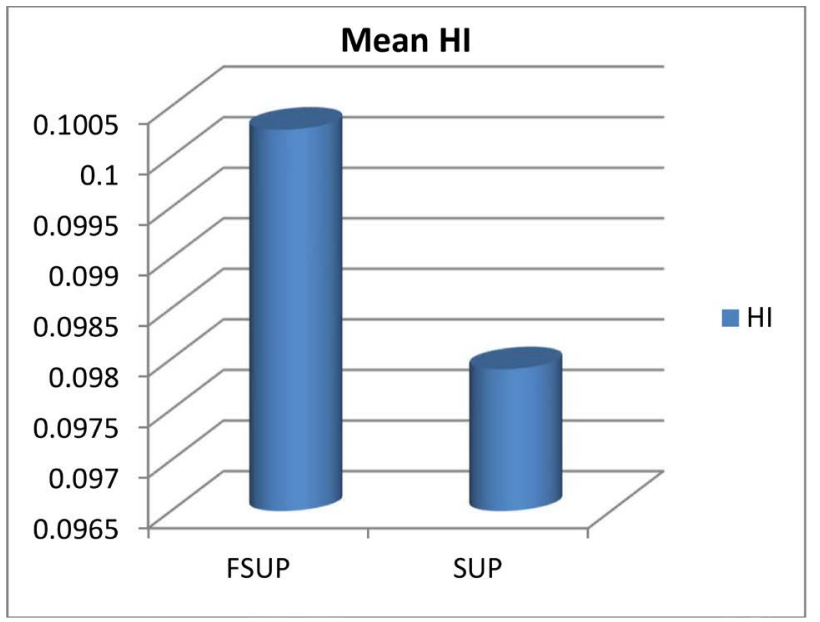

Figure 7. Comparison between two algorithms according to Homogeneity Index.

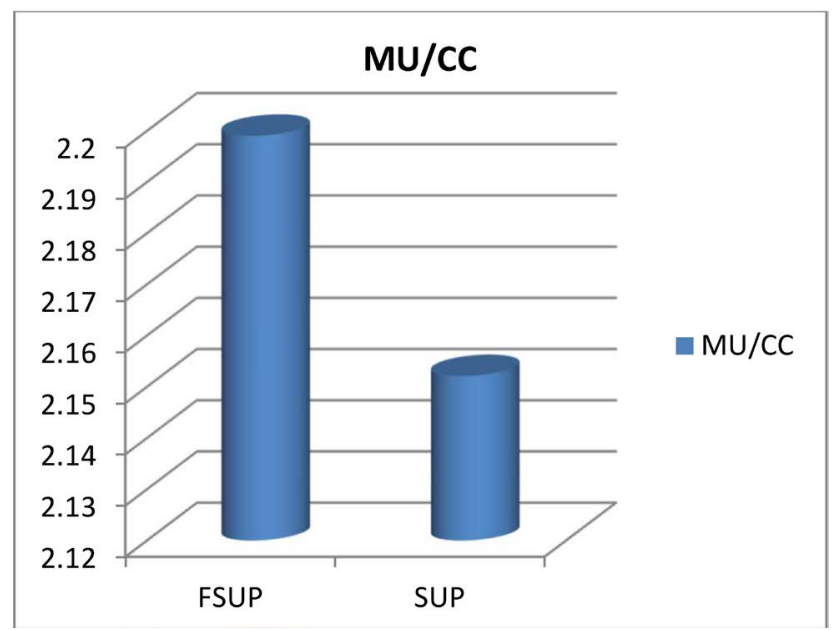

Figure 8. Comparison between two algorithms according to MU/CC with IMRT. 


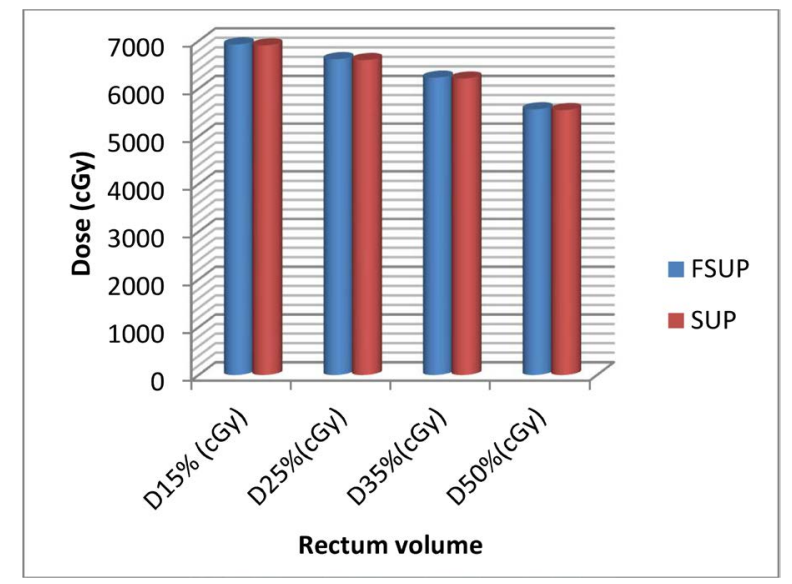

Figure 9. Comparison between two algorithms according to Rectum volume.

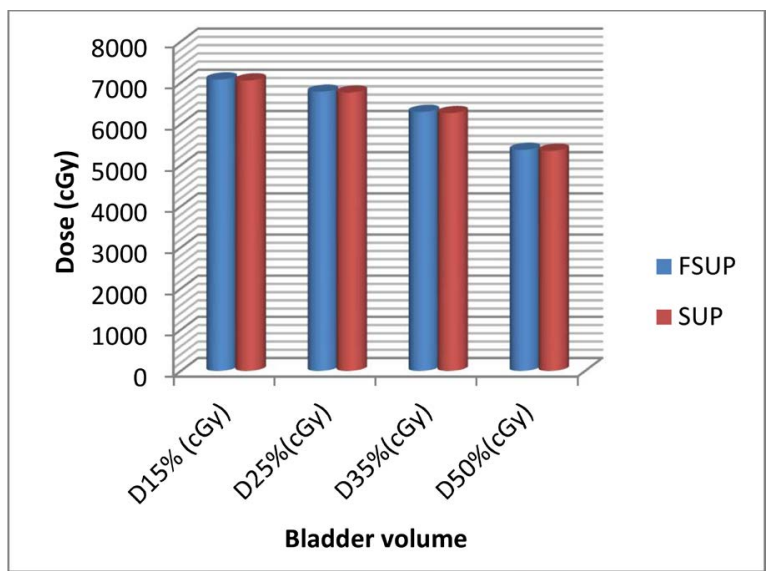

Figure 10. Comparison between two algorithms according to Bladder volume.

Table 3. D15\%, D25\%, D35\%, D50\% for rectum in both algorithm IMRT plan.

\begin{tabular}{|c|c|c|c|c|c|c|c|c|c|c|}
\hline \multirow{2}{*}{$\begin{array}{l}\text { Rectum } \\
\text { (cGy) }\end{array}$} & \multicolumn{4}{|c|}{ FSUP algorithm } & \multicolumn{4}{|c|}{ SUP algorithm } & \multicolumn{2}{|c|}{ PAIRED T TEST } \\
\hline & Mean & SD & Min. & Max. & Mean & SD & Min. & Max. & $\mathbf{t}$ & $\mathrm{p}$ \\
\hline $\mathrm{D} 15 \%$ & 6927.33 & 286.387 & 6368.2 & 7208 & 6907.02 & 274.925 & 6365.3 & 7186.6 & 3.45054 & $0.0073^{\star}$ \\
\hline D25\% & 6617.319 & 534.025 & 5741.8 & 7169.1 & 6599.852 & 522.864 & 5743.2 & 7156.9 & 2.38513 & $0.0409^{*}$ \\
\hline D35\% & 6231.19 & 784.957 & 5044.8 & 7112.3 & 6213.39 & 774.227 & 5048.4 & 7105.5 & 2.1897 & 0.0563 \\
\hline D50\% & 5566.99 & 1060.4 & 4104.8 & 6843.5 & 5549.33 & 1045.69 & 4111.8 & 6858.5 & 1.47997 & 0.173 \\
\hline
\end{tabular}

${ }^{*}$ Significant difference from all $(\mathrm{p} \leq 0.05)$.

Table 4. D15\%, D25\%, D35\%, D50\% for bladder in both algorithm IMRT plan.

\begin{tabular}{|c|c|c|c|c|c|c|c|c|c|c|}
\hline \multirow{2}{*}{$\begin{array}{c}\text { Bladder } \\
\text { (cGy) }\end{array}$} & \multicolumn{4}{|c|}{ FSUP algorithm } & \multicolumn{4}{|c|}{ SUP algorithm } & \multicolumn{2}{|c|}{ PAIRED T TEST } \\
\hline & Mean & SD & Min. & Max. & Mean & SD & Min. & Max. & $\mathrm{t}$ & $\mathrm{p}$ \\
\hline D15\% & 7078.33 & 152.54 & 6873.4 & 7275 & 7054.34 & 146.99 & 6846 & 7249 & 5.324 & $0.00048^{*}$ \\
\hline D25\% & 6780.93 & 454.25 & 5970.1 & 7232.7 & 6760.01 & 446.63 & 5968.3 & 7205.6 & 4.712 & $0.0011^{*}$ \\
\hline D35\% & 6290.05 & 877.58 & 4871.9 & 7193 & 6262.96 & 876.21 & 4899.1 & 7160 & 2.419 & $0.03868^{\star}$ \\
\hline D50\% & 5371.03 & 1255.4 & 3721 & 7095.5 & 5347.41 & 1232.8 & 3700 & 7038.2 & 2.582 & $0.02961^{*}$ \\
\hline
\end{tabular}

${ }^{*}$ Significant difference from all $(\mathrm{p} \leq 0.05)$. 
for FSP and 70.54 Gy, 67.61 Gy, 62.62 Gy, 53.47 Gy for SP algorithm for seven field IMRT Plans. As we can see the dose of all bladder volumes in the SP algorithm IMRT plan are less than that of the FSP algorithm.

However, statistically there were significant difference between them $(\mathrm{p}=$ $0.000, \mathrm{p}=0.001, \mathrm{p}=0.039 \mathrm{p}=0.030$ ).

Comparison between the FSUP and SUP algorithms for OARs (Femoral Head):

Figure 11, Figure $12 \&$ Table 5 shows the maximum and mean dose of right

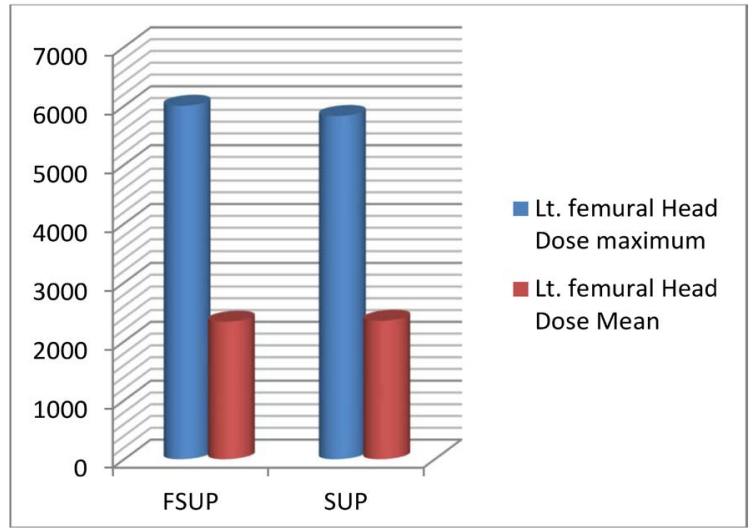

Figure 11. Comparison between two algorithms according to maximum\& mean dose for Lt. femoral head.

Table 5. Maximum and mean of the femoral head for both algorithms IMRT plan.

\begin{tabular}{|c|c|c|c|c|c|c|c|c|c|c|}
\hline \multirow{2}{*}{ Femoral Head } & \multicolumn{4}{|c|}{ FSUP algorithm } & \multicolumn{4}{|c|}{ SUP algorithm } & \multicolumn{2}{|c|}{ PAIRED T TEST } \\
\hline & Mean & SD & Min. & Max. & Mean & SD & Min. & Max. & $\mathbf{t}$ & $\mathrm{p}$ \\
\hline \multicolumn{11}{|l|}{ Rt. Femoral Head } \\
\hline Dose maximum & 5383.66 & 1301.17 & 3733.1 & 7073.3 & 5350.43 & 1278.27 & 3768.2 & 7038.7 & 1.9574 & 0.082 \\
\hline Dose Mean & 2274.7 & 689.67 & 1478.3 & 3386.1 & 2257.2 & 687.19 & 1460 & 3381.7 & 2.60582 & $0.028^{\star}$ \\
\hline \multicolumn{11}{|l|}{ Lt. Femoral Head } \\
\hline Dose maximum & 6000.16 & 1273.98 & 3485.5 & 6867.4 & 5827.25 & 1239.26 & 3540.5 & 6889.4 & 1.05255 & 0.320 \\
\hline Dose Mean & 2334.12 & 897.45 & 1257.8 & 3862 & 2350.38 & 884.88 & 1258 & 3950.7 & 0.43285 & 0.675 \\
\hline
\end{tabular}

${ }^{*}$ Significant difference from all $(\mathrm{p} \leq 0.05)$.

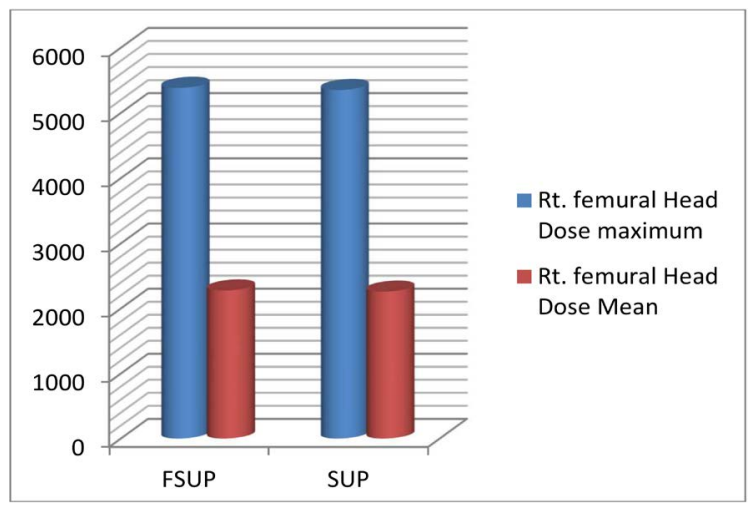

Figure 12. Comparison between two algorithms according to maximum \& mean dose for Rt. femoral head. 
\& Left Femoral Head for two algorithms. Maximum and mean dose of Lt. Femoral Head and Maximum dose of Rt. Femoral Head for both algorithms are non-significant difference were observed $(\mathrm{p}=0.320, \mathrm{p}=0.675, \mathrm{p}=0.082)$.

\section{Discussion}

In this study, adosimetric case study was performed for prostate cancer, and two algorithms (FSUP and SUP) IMRT plan were compared using the DVHs generated in the TPS. Rectum, which is extremely close to the PTV, is the most important organ at risk in prostate cancer. The table shows the dose to rectum D15, D25\% is significantly reduced in SUP algorithm IMRT plan. There is no significant difference was observed in both algorithm doses to rectum volume of D35\%, D50\%.

The bladder is another important organ that should be protected in prostate cancer treatment. SUP IMRT plan performed better plan quality on bladder sparing than FSUP IMRT plan at the dose of bladder volume D15\%, D25\%, D35\%, D50\%. However, we found that there was a significant variation between FSUP and SUP algorithm IMRT plan in bladder irradiated volume.

For delivery efficiency, our study showed that there is no significant difference in MU/CC for both algorithms.

\section{Conclusion}

We compared Fast superposition, Superposition algorithms (CMS, Xio treatment planning System) using seven field IMRT technique for prostate cancer. Significant variation was observed in maximum, mean, minimum doses of PTV for both algorithms. The superposition algorithm showed excellent results for prostate cancer. In our study, we recommend using of superposition algorithm with IMRT techniques in the treatment planning of prostate cancer. This recommendation is based on the better conformation of all PTV parameters and the sparing of the rectum, bladder, femoral head normal tissue.

\section{Ethical Approval}

This article does not contain any studies with human participants or animals performed by any of the authors.

\section{Conflicts of Interest}

The authors declare no conflicts of interest regarding the publication of this paper.

\section{References}

[1] Teoh, M., Clark, C.H., Wood, K., Whitaker, S. and Nisbet, A. (2011) Volumetric Modulated Arc Therapy: A Review of Current Literature and Clinical Use in Practice. British Journal of Radiology, 84, 967-996. https://doi.org/10.1259/bjr/22373346

[2] Hall, E.J. and Wuu, C.S. (2003) Radiation-Induced Second Cancers: The Impact of 
3D-CRT and IMRT. International Journal of Radiation Oncology ${ }^{\star}$ Biology ${ }^{\star}$ Physics, 56, 83-88. https://doi.org/10.1016/S0360-3016(03)00073-7

[3] Dearnaley, D.P., Sydes, M.R., Graham, J.D., et al. (2007) Escalated-Dose versus Standard-Dose Conformal Radiotherapy in Prostate Cancer: First Results from the MRC RT01 Randomised Controlled Trial. The Lancet Oncology, 8, 475-487. https://doi.org/10.1016/S1470-2045(07)70143-2

[4] Peeters, S.T., Heemsbergen, W.D., Koper, P.C., et al. (2006) Dose-Response in Radiotherapy for Localized Prostate Cancer: Results of the Dutch Multicenter Randomized Phase III Trial Comparing 68 Gy of Radiotherapy with 78 Gy. Journal of Clinical Oncology, 24, 1990-1996. https://doi.org/10.1200/JCO.2005.05.2530

[5] Zelefsky, M.J., Fuks, Z., Happersett, L., Lee, H.J., Ling, C.C., et al. (2000) Clinical Experience with Intensity Modulated Radiation Therapy (IMRT) in Prostate Cancer. Radiotherapy and Oncology, 55, 241-249. https://doi.org/10.1016/S0167-8140(99)00100-0

[6] Shu, H.K., Lee, T.T., Vigneauly, E., et al. (2001) Toxicity Following High-Dose ThreeDimensional Conformal and Intensity-Modulated Radiation Therapy for Clinically Localized Prostate Cancer. Urology, 57, 102-107. https://doi.org/10.1016/S0090-4295(00)00890-6

[7] Vanderstraeten, B., Reynaert, N., Paelinck, L., Madani, I., De Wagter, C., De Gersem, W., et al. (2006) Accuracy of Patient Dose Calculation nf or Lung IMRT: A Comparison of Monte Carlo, Convolution/Superposition, and Pencil Beam Computations. Medical Physics, 33, 3149. https://doi.org/10.1118/1.2241992

[8] Radiation Therapy Oncology Group (RTOG). Contouring Atlases: Breast Cancer Atlas. https://www.rtog.org/CoreLab/ContouringAtlases/BreastCancerAtlas.aspx

[9] Commission on Radiation Units and Measurements (2010) Measurements. Report83. Prescribing, Recording, and Reporting Photon-Beamintensity-Modulated Radiation Therapy (IMRT). Oxford University Press.

[10] Shaw, E., Kline, R., Gillin, M., Souhami, L., Hirschfeld, A., Dinapoli, R., et al. (1993) Radiation Therapy Oncology Group: Radiosurgery Quality Assurance Guidelines. International Journal of Radiation Oncology, Biology, Physics, 27, 1231-1239. https://doi.org/10.1016/0360-3016(93)90548-A 